\title{
Assistência obstétrica no trabalho de parto e parto em mulheres de risco habitual: revisão narrativa
}

\section{Obstetric assistance in labor and birth in women at common risk: narrative review}

\author{
Maxsuênia Queiroz Medeiros ${ }^{1}$. Débora Rodrigues Mesquita². Vivian Martins dos Santos Leite ${ }^{3}$. João Gabriel \\ Damasceno Pereira ${ }^{3}$. Francisco Herlânio Costa Carvalho ${ }^{4}$.
}

1 Mestre em Saúde Coletiva pela Universidade Federal do Ceará(UFC), Fisioterapeuta pela Maternidade Escola Assis Chateaubriand (MEAC), Fortaleza, Ceará, Brasil. 2 Graduada em Fisioterapia pela Faculdade de Tecnologia Intensiva (FATECI), Fortaleza, Ceará, Brasil. 3 Graduando(a) em Medicina pela Universidade Federal do Ceará (UFC), Fortaleza, Ceará, Brasil. 4 Doutor em Medicina, Professor Adjunto do departamento de Saúde Materno Infantil pela Universidade Federal do Ceará (UFC), Fortaleza, Ceará, Brasil.

\section{RESUMO}

O presente estudo teve como objetivo realizar uma revisão na literatura acerca da assistência obstétrica no trabalho de parto e parto em mulheres de risco habitual, com o intuito de investigar essa assistência no cenário mundial e nacional. Para esse fim, realizou-se uma revisão nas bases de dados eletrônicas SciELO, PUBMED e BIREME. A ruptura artificial das membranas, ou amniotomia, apesar de ser um procedimento simples, não é isenta de riscos. Não existem evidências capazes de embasar a sua prática, efetividade e segurança; não sendo, portanto, recomendada como método de indução do parto. Porém, os estudos analisados demostram uso considerável de amniotomia, com taxas de $40 \%$ a 53,4\%. Observou-se que a frequência de práticas desnecessárias, que devem ser evitadas e continuam sendo executadas de forma indiscriminada, ainda é significativa.

Palavras-chave: Assistência ao parto. Trabalho de parto. Parto humanizado.

\section{ABSTRACT}

The present study aims to review the literature on obstetric care in labor and delivery in women of normal risk, with the purpose of investigating this assistance in the world and national scenario. For this purpose, a review was carried out in the electronic databases SciELO, PUBMED and BIREME. Artificial rupture of the membranes, or amniotomy, although it is a simple procedure, is not without risk. There is no evidence capable of supporting its practice, effectiveness and safety; therefore, it is not recommended as a method of induction of labor. However, the studies analyzed show a considerable use of amniotomy, with a rate of $40 \%$ and $53.4 \%$. It has been observed that the frequency of unnecessary practices, which must be avoided and continue to be executed indiscriminately, is still significant.

Keywords: Midwifery. Labor, obstetric. Humanizing delivery.

Autor correspondente: Maxsuênia Queiroz Medeiro, Rua Diamante, 100, Mondubim, Fortaleza, Ceará. CEP: 60714-222. Telefone: +55 85 3493-6649. E-mail: maxsuenia@yahoo.com.br

Conflito de interesses: Não há qualquer conflito de interesses por parte de qualquer um dos autores.

Recebido em: 17 Mar 2017; Revisado em: 11 Jun 2017; Aceito em: 06 Ago 2017. 


\section{INTRODUÇÃO}

$\mathrm{O}$ cuidado prestado à mulher e seu concepto no momento do parto e nascimento passou por diversas modificações com o advento da institucionalização e medicalização na assistência em saúde. ${ }^{1}$ Essas transformações ocorreram em decorrência do salto do desenvolvimento técnico e científico nas práticas obstétricas e tiveram como consequência a retirada do protagonismo das mulheres no nascimento dos seus filhos.

O parto, antes visto como um processo fisiológico e natural, passou a ser considerado perigoso, necessitando da presença de um médico, além de sua assistência tornar-se cercada de procedimentos por vezes desnecessários e invasivos. ${ }^{2}$ No Brasil, a assistência obstétrica tem sido caracterizada pelo excesso de intervenções no parto, contribuindo para o aumento de taxas de cesáreas e da morbimortalidade materna e perinatal. ${ }^{3}$

Com o atual cenário de crescimento nas taxas de cesarianas, queda nos números de parto normal e excesso de intervenções desnecessárias, o mundo lançou olhar para tal questão, na tentativa de minimizar efeitos deletérios dessas práticas às parturientes e seus conceptos, apresentando evidências científicas para mudanças dessas práticas. ${ }^{4}$

Dentre as estratégias utilizadas para melhorar a assistência obstétrica o Ministério da Saúde, por meio de suas normas, passou a assegurar à parturiente o direito de um acompanhante durante o trabalho de parto, a escolha da posição para parir, medidas de controle da dor no período expulsivo e ao aleitamento materno na sala de parto..$^{5,6}$

A portaria que institui no âmbito do Sistema Único de Saúde a Rede Cegonha ${ }^{5}$ veio reforçar o que em 1996 foi preconizado pela Organização Mundial da Saúde (OMS) em seu Guia prático para Assistência ao Parto Normal, ressaltando que as práticas de atenção à saúde, "Boas práticas de atenção ao parto e ao nascimento", sejam baseadas em evidências científicas. Este documento ainda classifica a assistência obstétrica em quatro categorias: A, B, C e D (OMS, 1996). ${ }^{7}$

As práticas da Categoria $\mathrm{A}$, são práticas úteis e que devem ser estimuladas, exemplo delas são a liberdade de posição e movimento, métodos não-invasivos e não farmacológicos de alívio da dor e presença de acompanhante durante o trabalho de parto.

As práticas no parto normal claramente prejudiciais ou ineficazes e que devem ser eliminadas, são classificadas como práticas da Categoria $\mathrm{B}$, como uso rotineiro da posição de litotomia, toques vaginais frequentes e uso liberal ou rotineiro da episiotomia. A Categoria $\mathrm{C}$, diz respeito às práticas no parto normal para as quais não existem evidências para apoiar sua recomendação e que devem ser utilizadas com cautela até que novas pesquisas esclareçam a questão, como clampeamento precoce do cordão umbilical, pressão no fundo uterino no período expulsivo e estimulação do mamilo para aumentar a contratilidade uterina durante o terceiro estágio do parto.

Existem práticas no parto normal que são frequentemente utilizadas de modo inadequado, elas representam as práticas da Categoria $\mathrm{D}$, entre elas estão a restrição hídrica e alimentar durante o trabalho de parto, controle da dor por analgesia peridural e amniotomia precoce de rotina no primeiro estágio do parto.?

\section{METODOLOGIA}

O levantamento bibliográfico foi realizado nas bases de dados eletrônicas SciELO, PUBMED e BIREME no período de julho de 2015 a maio de 2016, utilizando os seguintes descritores: "Assistência ao parto", "Trabalho de parto", "Parto humanizado", em diversas combinações. Foram definidos como critérios de inclusão os estudos publicados nos últimos cinco anos em português, inglês e espanhol, com resumos disponíveis nas bases de dados selecionadas e que se adequassem ao objetivo desta revisão. Foram excluídos aqueles não disponibilizados na íntegra, bem como os artigos de revisão.

\section{RESULTADOS}

A partir da presente revisão foi possível perceber diversos avanços no que diz respeito à busca por uma assistência adequada, de acordo com as recomendações da OMS e do Ministério da Saúde.

A episiotomia ainda é prática frequente em diversos centros, assim como a infusão de ocitocina no primeiro e segundo estágios do trabalho de parto e a ruptura artificial das membranas, ou amniotomia.

Exemplo disso encontra-se no estudo retrospectivo realizado em Itapecerica da Serra em São Paulo, com objetivo de associar a integridade perineal, laceração espontânea e episiotomia em partos normais com a idade materna, paridade, idade gestacional, peso e vitalidade do recém-nascido com 6.365 partos, o qual demonstrou que $25,9 \%$ das mulheres foram submetidas ao procedimento. O estudo observou que a chance da realização de episiotimia aumentou com a nuliparidade $(\mathrm{OR}=3,0)$, prematuridade $(\mathrm{OR}=2,3)$ e Apgar inferior à 7 no primeiro minuto $(\mathrm{OR}=2,1)$, enquanto a chance de laceração de segundo grau associou-se com peso do recém-nascido superior à $3.300 \mathrm{~g}(\mathrm{OR}=1,6)$. Concluiu-se que houve associação do desfecho perineal com a paridade, prematuridade, peso e vitalidade do recém-nascido. ${ }^{8}$

Dahlen et al no ano de 2012 e Ballesteros-Meseguer et al em 2016 observaram em seus estudos que primíparas possuem maiores chances de realizarem episiotomia. ${ }^{9,10} \mathrm{Na}$ pesquisa de Dahlen, H. G, et al. de caráter descritivo de base populacional realizado em New South Wales, na Austrália contendo 691.738 mulheres, evidenciou-se que as primíparas de baixo risco que deram à luz em um hospital privado, quando comparadas às de um hospital público, tiveram maiores taxas de episiotomia ( $28 \%$ vs $12 \%$ ), enquanto nas multíparas de baixo risco envolvidas no estudo, esse percentual diminui para ( $8 \%$ vs $2 \%$ ). Ballesteros-Meseguer e colaboradores identificou que a taxa de episiotomias em mulheres primíparas, foi de $2.450(68,3 \%)$ em 2011 e 2.388 (70,3\%) em 2012; e entre mulheres com um ou 
mais partos anteriores (multíparas), o número de episiotomias foi de 783 (27,1\%) em 2011 e 653 (31\%) em 2012.

Embora essa prática, segundo o Ministério da Saúde, seja claramente prejudicial ou ineficaz, e que deveria ser eliminada, ainda existem instituições insistindo em seu uso, como foi observado no estudo realizado com 404 mulheres que avaliou partos normais em 13 hospitais de Goiânia, durante o período de abril a dezembro de 2007, com objetivo de avaliar a qualidade da assistência hospitalar ao parto normal, o qual constatou taxa de $70,1 \%$ de episiotomia. ${ }^{11}$ Dados semelhantes foram encontrados no estudo de Leal e colaboradores (2014) que identificou a adoção do método em $56 \%$ das mulheres envolvidas no estudo. ${ }^{12}$ Porcentagens menores podem ser observadas no estudo de Silva e colaboradores em 2013 e Chalmers et al em 2009 com uma taxa de $14,1 \%$ e $20,7 \%$, respectivamente. ${ }^{13,14}$

A ruptura artificial das membranas, ou amniotomia, apesar de ser um procedimento simples, não é isenta de riscos. Não existem evidências capazes de embasar a sua prática, efetividade e segurança; não sendo, portanto, recomendada como método de indução do parto. ${ }^{7}$ Porém, os estudos analisados demostram uso considerável de amniotomia, com uma taxa de $40 \%$ e 53,4\% nas pesquisas de Leal et al em 2014 e Silva et al em 2013, respectivamente. ${ }^{12,13}$

Quanto à realização de analgesia raqui/epidural, durante o trabalho de parto, foi possível identificar na Austrália, uma taxa de $53 \%$ das primíparas de baixo risco e $35 \%$ das multíparas que deram à luz em um hospital privado que receberam analgesia epidural. Assim como estudo de Chalmers et al $^{14}$ e Leal e colaboradores ${ }^{12}$ nos quais a analgesia foi executada, respectivamente, em $57,3 \%$ e $30 \%$ das mulheres estudadas.

O estímulo a posições não supinas durante o trabalho de parto faz parte das práticas da Categoria A, devendo, portanto, ser estimuladas. Entretanto, nos estudos pesquisados, observouse percentuais elevados de mulheres que pariram em posição litotômica, como em Goiânia, onde nenhum parto foi registrado em posição não-supina. Do mesmo modo Leal et al (2014) verificou uma taxa de $92 \%$ e Chalmers et al (2009) $)^{14} 47,9 \%$ de partos em posição de litotomia. Resultados diferentes dos encontrados no estudo de uma casa de parto na cidade de São Paulo, Brasil ${ }^{13}$, onde $82,3 \%$ das mulheres pariram na posição semi-sentada, posição preconizada por ser verticalizada.

Em pacientes de risco habitual não se recomenda realização de jejum e em pacientes de alto risco não há evidências suficientes também para sua recomendação. ${ }^{15}$ Não há necessidade do jejum rotineiro para parturientes de risco habitual. Singata, Tranmer e Gyte, ${ }^{16}$ em uma meta-análise com cinco estudos e 3.130 mulheres demonstraram que não existem resultados significantes que justifiquem tal prática nem mesmo quando correlacionaram a ingestão de alimentos ou líquidos com náuseas e vômitos maternos. Sendo, portanto, indicado que elas possam ser encorajadas a se comportar com liberdade e autonomia para escolher alimentar-se ou ingerir líquidos durante o trabalho de parto.

Entretanto, a restrição alimentar durante o trabalho de parto ainda se faz de forma rotineira em algumas instituições, como pode ser observado no estudo de Leal et al (2014) $)^{12}$ e Giglio et al (2011), ${ }^{11}$ nos quais menos de $1 / 3$ das parturientes se alimentaram e $62 \%$ estiveram em dieta zero durante o trabalho de parto, respectivamente. Diferentemente do resultado encontrado no estudo de Silva e colaboradores (2013), ${ }^{13}$ onde apenas 4,5\% das mulheres estudas não se alimentou durante o período.

A escolha de acompanhante, liberdade de movimentação e métodos não invasivos e não farmacológicos de alívio da dor, como massagens e técnicas de relaxamento durante o trabalho de parto, são práticas que devem ser estimuladas e fazer parte da rotina no trabalho de parto. No estudo de Silva e colaboradores (2013), ${ }^{13}$ 60\% das parturientes receberam massagem, 84\% banho de aspersão, $68 \%$ se movimentaram livremente e $51 \%$ realizaram exercícios em bolas suíças, podendo ser observado que os profissionais do centro de parto estudado realizam os métodos não invasivos e não farmacológicos para alívio da dor. Cenário diferente do encontrado no estudo de Leal et al (2014), ${ }^{12}$ onde menos de um terço das mulheres estudadas receberam tais técnicas e apenas $45 \%$ deambularam durante o trabalho de parto.

O partograma, instrumento utilizado para realizar o acompanhamento da evolução do trabalho de parto, possibilitando realizar o diagnóstico de possíveis alterações e indicação de condutas apropriadas para a sua resolução, é considerado como instrumento de uso indispensável. ${ }^{17}$ Todavia, seu uso ainda é pouco realizado, como visto nos estudos de Giglio et al (2011) ${ }^{11}$ e Leal e colaboradores (2014), ${ }^{12}$ onde apenas $28,5 \%$ e $45 \%$ das parturientes tiveram progresso monitorado no partograma, respectivamente.

\section{CONCLUSÃO}

Foi possível perceber com a presente revisão, que os centros de parto normal e unidades autônomas ou alternativas para parto apresentam menores taxas de intervenções obstétricas que as apresentadas nos hospitais. Ficou claro que a frequência de práticas desnecessárias, que devem ser evitadas e continuam sendo executadas de forma indiscriminada ainda é significativa, sendo representado pela elevada prevalência de intervenções desnecessárias compilados no presente estudo. Isso mantém a assistência à díade mãe-bebê aquém do que a Organização Mundial da Saúde preconiza, demonstrando ser esta uma problemática da saúde coletiva em um contexto mundial, para a qual é necessária atenção priorizada.

\section{REFERÊNCIAS}

1. Brasil. Ministério da Saúde. Humanização do parto e do nascimento. Brasília: Ministério da Saúde; 2014. 465 p. (Cadernos HumanizaSUS; v. 4).
2. Gomes K. Intervenções obstétricas realizadas durante o trabalho de parto e parto em uma maternidade de baixo risco obstétrico, na cidade de Ribeirão Preto [dissertação]. Ribeirão Preto: Escola de 
enfermagem de Ribeirão Preto; 2011. 142p.

3. Oreano JM, Brüggemann OM, Velho MB, Monticelli M. Visão de puérperas sobre a não utilização das boas práticas na atenção ao parto. Cienc Cuid Saude. 2014;13(1):128-36.

4. Organização Mundial de Saúde. Declaração da OMS sobre taxas de cesáreas. Genebra: OMS, 2015.

5. Brasil. Ministério da Saúde. Portaria n. ${ }^{\circ}$ 1.459, de 24 de junho de 2011. Institui, no âmbito do Sistema Único de Saúde (SUS), a Rede Cegonha. Brasília: Diário Oficial da União; 2011.

6. Prata KS, Brito MC, Almeida MS, Barbosa NR, Nunes IM. Acompanhante no centro obstétrico: significado para a equipe de enfermagem. Revista Baiana de Enfermagem. 2011; 25(3):277-86.

7. Organização Mundial da Saúde. Assistência ao parto normal: um guia prático. Genebra: OMS; 1996.

8. Riesco ML, Costa, AS, Almeida SF, Basile AL, Oliveira SM. Episiotomia, laceração e integridade perineal em partos normais: análise de fatores associados. Rev Enferm UERJ. 2011;19(1):77-83.

9. Dahlen HG, Tracy S, Tracy M, Bisits A, Brown C, Thornton C. Rates of obstetric intervention among low-risk women giving birth in private and public hospitals in NSW: a population-based descriptive study. BMJ Open. 2012;2(5):1723-31.

10. Ballesteros-Meseguer C, Carrillo-García C, Meseguer-dePedro M, Canteras-Jordana M, Martínez-Roche ME. Episiotomy and its relationship to various clinical variables that influence its performance. Rev Lat Am Enfermagem. 2016;24:e2793.
11. Giglio MR, França E, Lamounier JA. Avaliação da qualidade da assistência ao parto normal. Rev Bras Ginecol Obstet. 2011;33(10):297-304.

12. Leal M, Pereira AP, Domingues RM, Theme MM Filha, Dias MA, Nakamura-Pereira M, et al. Intervenções obstétricas durante o trabalho de parto e parto em mulheres brasileiras de risco habitual. Cad Saude Publica. 2014;30(Supl 1):S17-47.

13. Silva FM, Paixão TC, Oliveira SM, Leite JS, Riesco ML, Osava RH. Assistência em um centro de parto segundo as recomendações da Organização Mundial da Saúde. Rev Esc Enferm USP. 2013;47(5):1031-8.

14. Chalmers B, Kaczorowski J, Levitt C, Dzakpasu S, O’Brien $\mathrm{B}$, Lee $\mathrm{L}$, et al. Use for routine interventions in vaginal labor and birth: findingns from the maternity experiences survey. Birth. 2009;36(1):13-25.

15. Porto AM, Amorim MM, Souza AS. Assistência ao primeiro período do trabalho de parto baseada em evidências: [revisão]. Femina. 2010;38(10):527-37.

16. Singata M, Tranmer J, Gyte GM. Restricting oral fluid and food intake during labour. Cochrane Database Syst Rev. 2010;20(1):CD003930.

17. Guida NF, Lima GP, Pereira AL. O ambiente de relaxamento para humanização do cuidado ao parto hospitalar. Rev Min Enferm. 2013;17(3):531-7.

\section{Como citar:}

Medeiros MQ, Mesquita DR, Leite VM, Pereira JG, Carvalho FH. Assistência obstétrica no trabalho de parto e parto em mulheres de risco habitual: revisão narrativa. Rev Med UFC. 2018 abr-jun;58(2):47-50. 